\title{
An Accurate and Robust Finite Element Level Set Redistancing Method
}

\author{
Patrick Esser $^{\dagger}$ and Jörg Grande ${ }^{\ddagger}$
}

Bericht Nr. 379

November 2013

Key words: level set method, redistancing, reinitialization

AMS Subject Classifications: 65M06, 65D05

Institut für Geometrie und Praktische Mathematik RWTH Aachen

Templergraben 55, D-52056 Aachen (Germany)

\footnotetext{
$†$ Email: pesser@igpm.rwth-aachen.de

† Corresponding author. Email: grande@igpm.rwth-aachen.de
} 


\title{
An Accurate and Robust Finite Element Level Set Redistancing Method
}

\author{
PATRICK Esser $†$ AND JÖRG GRANDEł \\ Institut für Geometrie und Praktische Mathematik, \\ RWTH Aachen University, Templergraben 55, D-52056 Aachen, Germany
}

[Received on 15 December 2013]

\begin{abstract}
A new redistancing method for piecewise polynomial finite element level set functions is introduced. The method directly computes the distance to the implicitly given discrete level set. Rigorous error bounds and numerical experiments are provided. Both show that, up to constants, the method is as good as the nodal interpolation of the computationally unavailable signed distance function of the continuous level set.
\end{abstract}

Keywords: level set method; redistancing; reinitialization.

\section{Introduction}

This paper is concerned with implicitly defined (hyper-) surfaces $\Gamma_{h} \subset \mathbb{R}^{n}$ which are given as the zero level of a level set function $\varphi_{h}$. More specifically, an algorithm is presented and analyzed which computes an approximate signed distance function to $\Gamma_{h}$. This problem is called redistancing. It has applications to many geometric problems, for example, to path planning, Kimmel \& Sethian (2001). A major application of redistancing is in the level set method, when the latter is applied to the time evolution of implicitly defined free surfaces and phase-interfaces in numerical continuum mechanics, Sussman et al. (1994); Osher \& Fedkiw (2001); Sethian (1999). Most level set methods do not conserve the approximate signed-distance property, which is therefore periodically restored by redistancing. In this context, redistancing is also called reinitialization. Most redistancing methods in the literature can broadly be classified as either 'PDE-based' (Sethian, 1999, Chp. 11), Sussman et al. (1994); Hartmann et al. (2010) or 'geometry-based' Sethian (1996, 1999); Kimmel \& Sethian (1998); Chopp (2001); Mut et al. (2006); Reusken (2013). A more extensive comparison and further references can be found in Ausas et al. (2011).

As input to the redistancing method, the discrete level set function $\varphi_{h}$ is assumed to be a function from a finite element space $\mathbb{X}_{h}$ on a triangulation $\mathcal{T}_{h}$. It suffices to know $\varphi_{h}$ in a small neighborhood of $\Gamma_{h}$. For piecewise quadratic and higher order level set functions, it is in general difficult to obtain an explicit representation of $\Gamma_{h}$. Similarly, the signed distance function $d_{h}$ of $\Gamma_{h}$ is generally not an element of $\mathbb{X}_{h}$. The output of a redistancing method is a finite element function $\tilde{d}_{h} \in \mathbb{X}_{h}$ satisfying two conditions: It approximates a signed distance function, and its zero level $\tilde{\Gamma}_{h}$ approximates $\Gamma_{h}$. These goals are not compatible in the sense that one can almost never improve on both at the same time.

$\dagger$ Email: pesser@igpm.rwth-aachen.de.

${ }_{\ddagger}^{\ddagger}$ Corresponding author. Email: grande@igpm.rwth-aachen.de. 
The new method in this paper is geometry-based with the typical structure of a fastmarching method - it is split in an initialization phase and an extension phase. In the former, the value of $\tilde{d}_{h}$ is computed for all degrees of freedom in a neighborhood of $\Gamma_{h}$. From these values, $\tilde{d}_{h}$ is computed in the extension phase in the remaining parts of the domain. The initialization phase completely determines the new zero level $\tilde{\Gamma}_{h}$, in particular, its approximation quality. Therefore, a detailed discussion of the extension phase is not presented here; any of the methods already available for fast marching can be used.

The simple idea of the new method is to compute $\tilde{d}_{h}$ as the nodal interpolant of $d_{h}$ in a neighborhood of $\Gamma_{h}$ up to floating point precision. As the base case, one considers $\varphi_{h}$ on a single simplex $T$, where it is a single polynomial. The surface $\Gamma_{h} \cap T$ is a patch of the (global) zero level $\mathcal{V}$ of this polynomial. Let $x$ be an arbitrary point; later, $x$ is taken as a finite element node. The points on $\mathcal{V}$ which have extremal distance to $x$ are determined by a system of polynomial equations, which results from the Lagrange multiplier rule. For a linear or quadratic polynomial, the system of polynomial equations can be reduced to a single polynomial equation in one indeterminate, for which efficient and robust root finding algorithms are known in the literature. With this computation as base case, one can exactly compute the distance of $x$ to the surface patch $\Gamma_{h} \cap T$, and finally to $\Gamma_{h}$.

In the analysis, the discrete level set function $\varphi_{h}$ is viewed as an approximation of a continuous counterpart $\varphi$, which is not explicitly available to the redistancing method. Its zero level is denoted as $\Gamma$. Connected to $\Gamma$ is its signed distance function $d$, also not available to the redistancing method. The approximate signed-distance property of $\tilde{d}_{h}$ is quantified by a bound on

$$
\left\|\tilde{d}_{h}-d_{h}\right\|_{L^{\infty}\left(\Omega_{h}^{\Gamma}\right)}=\max \left\{\left|\tilde{d}_{h}(x)-d_{h}(x)\right| \mid x \in \Omega_{h}^{\Gamma}\right\}
$$

where $\Omega_{h}^{\Gamma}$ is a small common neighborhood of $\tilde{\Gamma}_{h}$ and $\Gamma_{h}$. Another interesting error measure is the approximation-error with respect to $d$ as measured by $\max \left\{\left|\tilde{d}_{h}(x)-d(x)\right| \mid x \in \Omega_{h}^{\Gamma}\right\}$. How well $\tilde{\Gamma}_{h}$ approximates $\Gamma_{h}$ and $\Gamma$, is measured by the Hausdorff-distances $\operatorname{dist}\left(\tilde{\Gamma}_{h}, \Gamma_{h}\right)$ and $\operatorname{dist}\left(\tilde{\Gamma}_{h}, \Gamma\right)$ between the zero levels. All these measures should be 'small' in a good redistancing method. The main result of the analysis, Theorem 3.2, is a $\mathcal{O}\left(h^{l+1}\right)$-bound on these errors, if the space $\mathbb{X}_{h}$ contains the piecewise polynomials of degree up to $l$ and if $\varphi_{h}$ approximates a level set function $\varphi$ which is sufficiently smooth in a small neighborhood of $\Gamma$.

Numerical experiments for piecewise quadratic level set functions complement the analytical results. A smoothly embedded surface is considered to demonstrate that Theorem 3.2 is a sharp result. Further examples, one with discontinuous curvature and one with (curved) edges, are considered, which show that the method is robust. By this, we mean that it does not fail on coarse meshes and that the error close to the smooth parts of the domain is still bounded as in Theorem 3.2.

The performance of redistancing methods known in the literature is typically evaluated only in numerical studies by applying them to certain model problems. Heuristic arguments and partial analyses are sometimes supplied. The only rigorous error analysis of a redistancing method that we are aware of is in the recent paper Reusken (2013). We compare the method from Reusken (2013) with our method. Both work in any number of space dimensions and are implemented in 3D for piecewise linear and piecewise quadratic finite elements. The generalization to higher polynomial degrees is more straightforward for Reusken (2013). The analysis in Reusken (2013) is technical and excludes some 'non-generic' geometric constellations. Our 
analysis is quite elementary. The method in Reusken (2013) is iterative, which may lead to convergence problems on coarse meshes. Our method works on coarse meshes, cf. Section 5.

The remainder of the paper is structured as follows. In Section 2, the initialization phase is described in detail. The error analysis is given in Section 3. Section 4 contains some details of the implementation which is used for the numerical experiments in Section 5.

\section{The initialization phase}

In the initialization phase, the value of $\tilde{d}_{h}$ is computed for all degrees of freedom of the finite element space $\mathbb{X}_{h}$ which lie in the direct neighborhood triangulation

$$
\mathcal{T}_{h}^{\Gamma}=\left\{S \in \mathcal{T}_{h} \mid S \cap \Gamma_{h} \neq \emptyset\right\}
$$

of the discrete zero level $\Gamma_{h}$. The space $\mathbb{X}_{h}$ is the space of continuous, piecewise polynomial finite elements of degree 1 or 2 with the set of standard nodal basis points denoted by $\mathcal{N}_{h}$. Spaces containing polynomials of higher degree are addressed in Remark 2.1. To simplify the presentation, it is assumed that $\Gamma_{h}$ is compact and does not meet $\partial \Omega$.

In all points $x \in \mathcal{N}_{h}$, the sign of $\tilde{d}_{h}(x)$ is set equal to the sign of $d_{h}(x)$, and the absolute value is determined as described in the following sub-sections. This completely determines $\tilde{\Gamma}_{h}=\left\{x \mid \tilde{d}_{h}(x)=0\right\}$ on $\mathcal{T}_{h}^{\Gamma}$.

\subsection{The distance to the zero level of a polynomial}

On any $n$-simplex $S \in \mathcal{T}_{h}^{\Gamma},\left.\varphi_{h}\right|_{S}$ is a polynomial $\varphi_{S}(y)=y^{T} A y+a^{T} y+a_{0}$ with coefficients $A \in \mathbb{R}^{n \times n}, a \in \mathbb{R}^{n}, a_{0} \in \mathbb{R}$. The matrix $A$ is symmetric. The notation $|\cdot|$ is used for the absolute value of scalars, the Euclidean vector norm, and the spectral norm of matrices. Let $x \in \mathbb{R}^{n}$ be an arbitrary point; later, $x$ is taken from $\mathcal{N}_{h}$. The distance from $x$ to $\Gamma_{S}=\left\{z \in \mathbb{R}^{n} \mid \varphi_{S}(z)=0\right\}$ is computed. To this end, a point $y \in \Gamma_{S}$ with

$$
|y-x|=\operatorname{dist}\left(x, \Gamma_{S}\right):=\inf \left\{|z-x| \mid z \in \Gamma_{S}\right\}
$$

is determined. It may be that $y$ is not contained in $S$. But as $\Gamma_{S}$ is a closed set, its intersection with a sufficiently large ball around $x$ is compact, which implies that the infimum is attained at some point $y$.

Two standard facts from linear algebra are used. The cofactor matrix $C$ of a matrix $M \in$ $\mathbb{R}^{n \times n}$ has the entries $C_{i j}=(-1)^{i+j} \operatorname{det} M_{\hat{i} \hat{j}}$, where $M_{\hat{i} \hat{j}}$ is obtained from $M$ by removing row $i$ and column $j$. The coefficients of $C$ are therefore polynomials of degree $n-1$ in the coefficients of $M$. Furthermore, there is a close connection to the inverse of $M: C^{T} M=M C^{T}=\operatorname{det}(M) I_{n \times n}$.

One minimizes $|y-x|, x \in \mathbb{R}^{n}$, under the constraint $\varphi_{S}(y)=0$. As $|y-x|$ and $|y-x|^{2}$ assume their minima for the same values of $y$, one can use the Lagrange-function

$$
L(y, \mu)=|y-x|^{2}+\mu \varphi_{S}(y)
$$

with the real-valued Lagrange multiplier $\mu$. This is a polynomial of degree two in $y$. By the Lagrange-multiplier-rule, necessary conditions for a minimizer under the given constraint are

$$
\begin{aligned}
& 0=D_{y} L(y, \mu)=-2 x+2 y+\mu(2 A y+a)=: 2 M_{\mu} y-2 x_{\mu}, \\
& 0=D_{\mu} L(y, \mu)=\varphi_{S}(y)
\end{aligned}
$$


with $M_{\mu}=I+\mu A$ and $x_{\mu}=x-\frac{\mu}{2} a$. Let $C_{\mu}$ be the cofactor-matrix of $M_{\mu}$. The first equation implies

$$
\operatorname{det} M_{\mu} y=C_{\mu}^{T} x_{\mu} .
$$

Multiplying the second equation by $\left(\operatorname{det} M_{\mu}\right)^{2}$ and inserting the previous equation yields

Lemma 2.1 If $y$ minimizes $|y-x|$ under $\varphi_{S}(y)=0$, then

$$
p(\mu):=x_{\mu}^{T} C_{\mu} A C_{\mu}^{T} x_{\mu}+\operatorname{det} M_{\mu} a^{T} C_{\mu}^{T} x_{\mu}+\left(\operatorname{det} M_{\mu}\right)^{2} a_{0}=0 .
$$

Equation (2.3) involves a polynomial of degree $2 n$ in $\mu$. Its real roots are computed. To obtain candidate-solutions $y$ of (2.2), in the generic case, the linear system in (2.2) is solved for each root $\mu$.

In exceptional cases, a root of (2.3) might be such that $M_{\mu}$ is a singular matrix, that is, there exists a non-zero vector $v$ with $M_{\mu} v=0$. This condition is checked with the help of the the Schur-decomposition $A Q=Q \Lambda$, where $\Lambda=\operatorname{diag}\left(\lambda_{1}, \ldots, \lambda_{n}\right)$ is diagonal and $Q=\left(q_{1}, \ldots, q_{n}\right)$ is orthogonal. As $M_{\mu}=I+\mu A$, there holds $M_{\nu} v=0$, if and only if $\mu \lambda_{i}=-1$ and $v$ is a non-zero multiple of $q_{i}$ for some $i \in\{1, \ldots, n\}$. The solution-set of (2.3) might contain such values for $\mu$, if $x$ lies on a symmetry axis of the conic. More precisely, there are 2 possibilities: First, $M_{\mu} y=x_{\mu}$ could be an inconsistent linear system, which can be checked easily using that $Q$ diagonalizes $M_{\mu}$. In this case, there is no solution to the linear system associated to $\mu$.

Second, $M_{\mu} y=x_{\mu}$ could be a consistent linear system, which implies that the solutions $y$ form an affine subspace $H$ of dimension $m$. Here, $m$ is the multiplicity of the eigenvalue $\lambda_{i}$ of $A$ with $\mu \lambda_{i}=-1$. If $m=n$, the level sets of $\varphi_{S}$ are spheres, the radius of which can easily be computed. Otherwise, $m<n$ and we obtain a dimensional reduction to $H$. In this case, the distance can be computed with the method in Subsection 2.2.

Remark 2.1 (Other polynomial degrees) If $\varphi_{S}$ is a linear polynomial, then $M_{\mu}=I$. Thus, $y=x_{\mu}$, which can be inserted into $\varphi_{S}\left(x_{\mu}\right)=0$. This yields a uniquely solvable linear equation because the coefficient of $\mu$ is $a^{T} a$.

The method is applicable for higher polynomial degrees. It requires the solution of (2.2), which remains a system of polynomial equations, but in which the first equation is now nonlinear in $y$. In analogy to the derivation of (2.3) one could use elimination theory with Groebner bases, cf. Cox et al. (1997). For the latter, the authors do not know of robust floating-point algorithms. Alternatively, one could directly apply an iterative root finder to (2.2).

\subsection{Higher codimension}

Suppose that the extremizer $y$ is restricted to the affine space $H$ given as the image of $l(z)=$ $B z+b$ with $B \in \mathbb{R}^{n \times m}, 1 \leqslant m<n, m=\operatorname{rank}(B)$, and $b \in \mathbb{R}^{n}$. This arose in some exceptional cases in the previous sub-section. This situation also arises, if the distance to $\Gamma_{S} \cap F$ is computed for an $m$-face of $S$.

Remark 2.2 One could proceed as before, and introduce a second Lagrange-multiplier $\tilde{\mu} \in \mathbb{R}$ for the new constraint,

$$
L(y, \mu, \tilde{\mu})=|y-x|^{2}+\mu \varphi_{S}(y)+\tilde{\mu} \tilde{l}(y),
$$

where $\tilde{l}$ is an implicit equation for the hyperplane. This approach seems quite elegant, however, when explicating the details, the second Lagrange-multiplier introduces new possible degeneracies. Also, due to the elimination of $\tilde{\mu}$, the degree of the polynomial equation for $\mu$ increases. 
The reduction to $H$ is performed explicitly by introducing orthogonal coordinates in the domain of $l$ which map to orthogonal coordinates of $H$. Let $A=Q R$ be a QR-decomposition of $A$ and denote by $\hat{Q}$ the orthogonal $n \times m$ matrix composed of the first $m$ columns of $Q$. This defines the coordinate system. The implicit quadratic equation for $\Gamma_{S} \cap H$ in these coordinates is

$$
\varphi_{H}(z)=\varphi_{S}(\hat{Q} z+b)=z^{T} \hat{Q}^{T} A \hat{Q} z+\left(a^{T} \hat{Q}+2 b^{T} A \hat{Q}\right) z+b^{T} A b+a^{T} b+a_{0} .
$$

Let $x_{H}$ be the orthogonal projection of $x$ onto $H$, which can be written as

$$
x_{H}=\hat{Q} \hat{z}+b, \quad \hat{z} \in \mathbb{R}^{m} \text {. }
$$

The distance between $x_{H}$ and an arbitrary point $\hat{Q} z+b$ in $H$ is

$$
\left|x_{H}-(\hat{Q} z+b)\right|=|\hat{Q}(\hat{z}-z)|=|\hat{z}-z| \text {. }
$$

This proves

LEMma 2.2 Let $z \in \mathbb{R}^{m}$ be the minimizer of $|\hat{z}-z|$ under the constraint $\varphi_{H}(z)=0$. Then, $y=\hat{Q} z+b$ minimizes $|x-y|$ under the constraints $\varphi_{S}(y)=0, y \in H$. The minimal distance $d$ satisfies $d^{2}=|\hat{z}-z|^{2}+\left|x-x_{H}\right|^{2}$.

The constrained minimization problem in Lemma 2.2 is the same as the one considered in Subsection 2.1 with $n$ replaced by $m$ and $\varphi_{S}$ replaced by $\varphi_{H}$. Hence, it can be solved as explained there.

\subsection{The distance with respect to a single $n$-simplex}

Let $S \in \mathcal{T}_{h}^{\Gamma}$ be an $n$-simplex (which intersects $\Gamma_{h}$ ), and consider the local signed distance function $d_{h}^{S}$ with

$$
\left|d_{h}^{S}(x)\right|=\min \left\{|x-y| \mid y \in \Gamma_{h} \cap S\right\}, \quad \operatorname{sgn}\left(d_{h}^{S}(x)\right)=\operatorname{sgn}(\varphi(x)) .
$$

The value $\left|d_{h}^{S}(x)\right|$ is computed as follows; a description in pseudo-code is Algorithm 1. The minimum in (2.4) is assumed in the interior of $S$ or on the boundary $\partial S$. The candidates for the minima in the interior are computed as in Subsection 2.1. The minima on $\partial S$ are computed by considering each of the $(n-1)$-faces $F$ of $S$ together with the embedding of $F$ in an affine space $H$ of dimension $n-1$. We use the method of Subsection 2.2 to compute candidates for the distance minimizers in the (relative) interior of $F$. The boundary extrema on $\partial F$ are computed by noting that the boundary of $F$ is composed of $(n-2)$-simplexes. One obtains a recursive scheme, in which the method from Subsection 2.1 is applied on all $m$-faces of $S, 0<m \leqslant n$. The recursion terminates for the vertices $v$ of $S$, for which $\left|d_{h}^{v}(x)\right|=|x-v|$.

\subsection{The output of the initialization phase}

Let $x \in \mathcal{N}_{h}$ be a degree of freedom of $\mathbb{X}_{h}$ which is contained in

$$
\Omega_{h}^{\Gamma}=\cup\left\{S \in \mathcal{T}_{h}^{\Gamma}\right\} .
$$




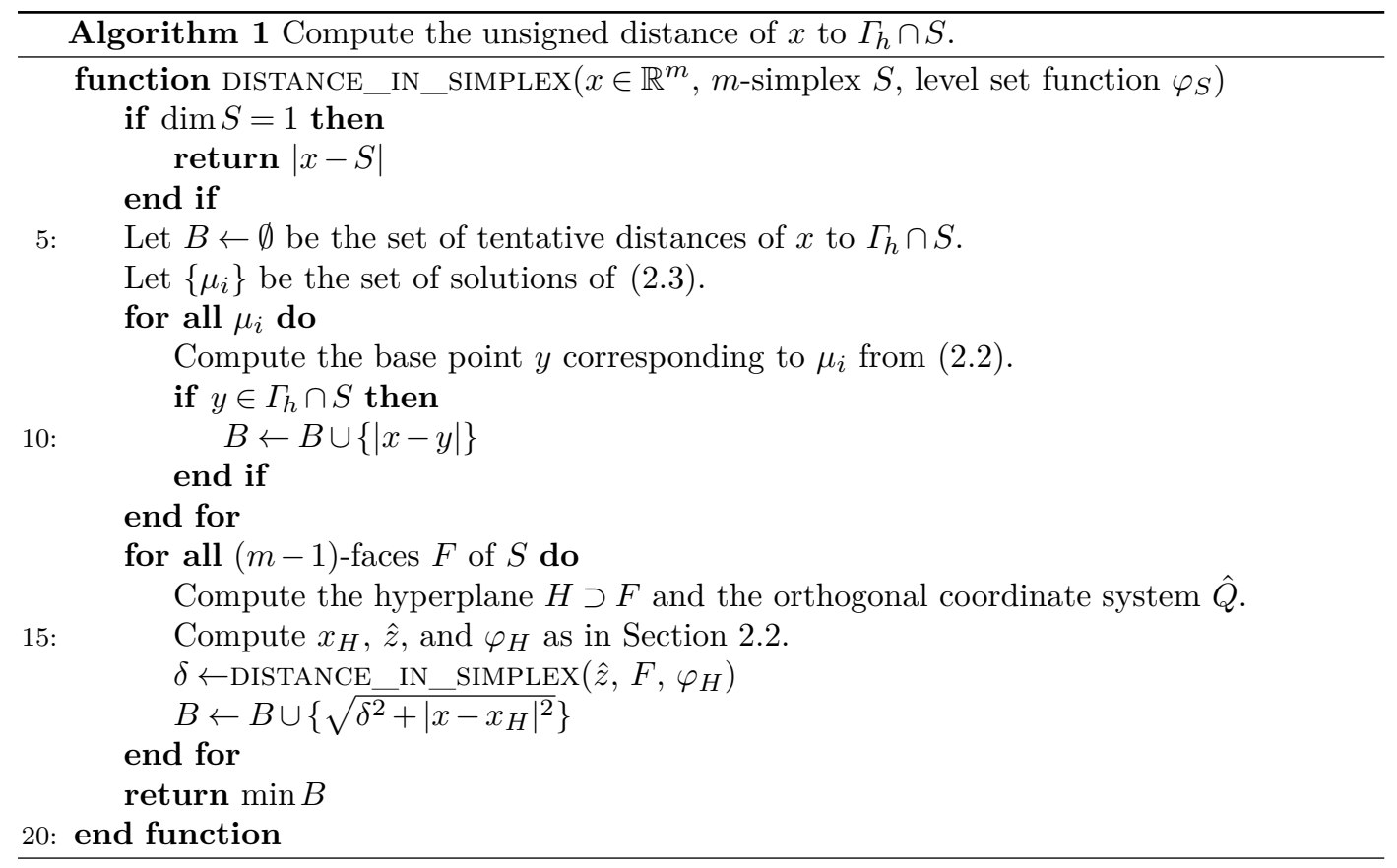

To evaluate $d_{h}(x)$, one uses $d_{h}^{S}$ from the previous section. An obvious consequence of $\Gamma_{h} \subset \Omega_{h}^{\Gamma}$ is

$$
\left|d_{h}(x)\right|=\min \left\{\left|d_{h}^{S}(x)\right| \mid S \in \mathcal{T}_{h}^{\Gamma}\right\} .
$$

The sign of $d_{h}(x)$ is set to the sign of $\varphi_{h}(x)$. One defines the output of the initialization phase as the function $\left.\tilde{d}_{h} \in \mathbb{X}_{h}\right|_{\Omega_{h}^{\Gamma}}$ which interpolates $d_{h}$,

$$
\tilde{d}_{h}(x)=d_{h}(x) \text { for all degrees of freedom } x \in \mathcal{N}_{h} \cap \Omega_{h}^{\Gamma} .
$$

To reduce the numerical work, it is desirable to take the minimum in (2.6) over a smaller neighborhood of $x$. As $x \in \Omega_{h}^{\Gamma}$, one of the $n$-simplices in

$$
\mathcal{T}^{1}(v)=\left\{S \in \mathcal{T}_{h} \mid v \in S\right\},
$$

has an intersection with $\Gamma_{h}$. Denote this simplex as $S$. Clearly, there holds $\left|d_{h}(x)\right| \leqslant\left|d_{h}^{S}(x)\right|$. Hence, it suffices to consider only the simplices of $\mathcal{T}_{h}^{\Gamma}$ which intersect the closed ball $B\left(x,\left|d_{h}^{S}(x)\right|\right)$. Furthermore, the upper bound $\left|d_{h}^{S}(x)\right|$ cannot be larger than $r_{x}=\max \{|x-y| \mid y \in T, T \in$ $\left.\mathcal{T}^{1}(x)\right\}$, which is a quantity that only depends on the geometry of the triangulations. For uniform triangulations (in the neighborhood of $\Gamma_{h}$ ), there is a fixed positive integer $k$ such that the ball $B\left(x, r_{x}\right)$ is covered by some

$$
\mathcal{T}^{k}(x)=\left\{S \in \mathcal{T} \mid S \cap T \neq \emptyset \text { for some } T \in \mathcal{T}^{k-1}(x)\right\} .
$$

For the numerical experiments in Section 5, we use triangulations, which are 3D-Kuhn triangulations in a neighborhood of $\Gamma_{h}$. A 2D-example is shown in Figure 1; the dots indicate all 

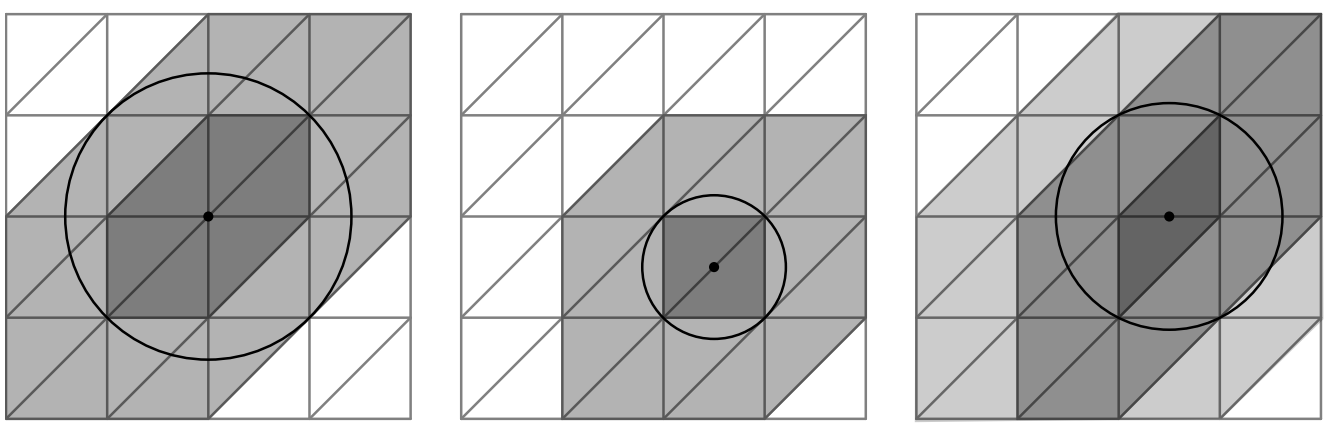

FIG. 1. The neighborhoods $\mathcal{T}^{k}$ on a regular 2D-mesh for a vertex (left, $k=2$ ) and the two types of edgebarycenters (middle and right, $k=2$ and $k=3$ ).

possible locations for the points in $\mathcal{N}_{h}$. One can see (and check by a few distance computations) that $k=3$ is the right choice to guarantee

$$
\left|d_{h}(x)\right|=\min \left\{\left|d_{h}^{S}(x)\right| \mid S \in \mathcal{T}^{k}(x)\right\}
$$

for all degrees of freedom $x \in \mathcal{N}_{h} \cap \Omega_{h}^{\Gamma}$. The choice $k=3$ also works on Kuhn-triangulations in 3D. The number of simplices in $\mathcal{T}^{k}$ for any fixed $k$ is bounded on quasi-uniform meshes. Hence, assuming $(2.8)$, one can evaluate $\tilde{d}_{h}(x)$ with a bounded number of local distance computations independent of $h$.

\section{Error analysis of the initialization phase}

The following assumptions on $\Gamma=\{x \in \Omega \mid \varphi(x)=0\}$ are used in the sequel: There is an open neighborhood $\Gamma \subset U \subseteq \Omega$ with $\varphi \in C^{k+1}(U)$ for some integer $k \geqslant 1$. The non-degeneracy of $\varphi$ is required as

$$
0<c_{0} \leqslant|D \varphi(x)| \leqslant c_{1} \quad \text { for all } x \in U
$$

with positive constants $c_{0}, c_{1}$. The real number $\max \left\{\left|D^{2} \varphi(x)\right| \mid x \in \bar{U}\right\}$ is denoted by $\tilde{\kappa}$; it is a measure of the curvature of $\varphi$.

Due to (Gilbarg \& Trudinger, 1977, App. A) and reducing $U$ if necessary, the signed distance function of $\Gamma$ satisfies $d \in C^{k+1}(U)$; the sign is chosen to be equal to the sign of $\varphi$. The gradient of $d$ is a unit-length normal field $\mathbf{n}=D d$ defined on $U$. It is assumed that $U$ is tubular, which means that there is a function $p: U \rightarrow \Gamma$ describing the perpendicular base point on the surface with the unique decomposition

$$
x=d(x) \mathbf{n}(p(x))+p(x) \quad \text { for all } x \in U .
$$

The function $p$ is in $C^{k}(U)$. The Hessian of $d$ is denoted by $H=D^{2} d$. The spectral norm $|H(x)|$ is a measure of the curvature of the level set of $d$ passing through $x$; let $\kappa=\max \{|H(x)| \mid x \in \Gamma\}$ be the maximal curvature of $\Gamma$. In fact, there holds

Lemma 3.1 For all $x \in U$ with $|d(x)| \leqslant \frac{1}{2 \kappa}$, there holds $|H(x)| \leqslant 2 \kappa$. 
Proof. From differentiating (3.2) and elementary rearrangements, one finds

$$
H(x)=H(\xi)(1+d(x) H(\xi))^{-1} \quad \text { for all } x \in U, \xi=p(x) .
$$

Applying the spectral theorem, one obtains the relation

$$
\lambda_{i}(x)=\frac{\lambda_{i}(\xi)}{1+d(x) \lambda_{i}(\xi)}, \quad i \in\{1, \ldots, n\},
$$

between the eigenvalues of $H(x)$ and $H(\xi)$. For distances $|d(x)| \leqslant(2 \kappa)^{-1}$, one has

$$
\left|\lambda_{i}(x)\right| \leqslant \frac{\left|\lambda_{i}(\xi)\right|}{1-|d(x)|\left|\lambda_{i}(\xi)\right|} \leqslant 2\left|\lambda_{i}(\xi)\right| .
$$

LEMma 3.2 For all $x \in U$ with $|d(x)| \leqslant \min \left\{\frac{1}{2 \kappa}, \frac{1}{2} \frac{c_{0}}{\tilde{\kappa}+2 \kappa c_{1}}\right\}$, there holds $\mathbf{n}(x)^{T} D \varphi(x) \geqslant \frac{c_{0}}{2}$.

Proof. Consider two arbitrary points $x, \xi \in U$ such that the line segment between them also is in $U$. The Taylor expansion of $\mathbf{n}(x)^{T} D \varphi(x)$ yields

$$
\mathbf{n}(x)^{T} D \varphi(x)=\mathbf{n}(\xi)^{T} D \varphi(\xi)+D\left(\mathbf{n}(\zeta)^{T} D \varphi(\zeta)\right)^{T}(x-\xi)
$$

with some $\zeta$ in the open line segment between $\xi$ and $x$. From $D\left(\mathbf{n}^{T} D \varphi\right)=\mathbf{n}^{T} D^{2} \varphi+H D \varphi$ and Lemma 3.1, one computes

$$
\left|D\left(\mathbf{n}(\zeta)^{T} D \varphi(\zeta)\right)\right| \leqslant \tilde{\kappa}+2 \kappa c_{1} .
$$

Now choose $\xi=p(x) \in \Gamma$. Hence, $\mathbf{n}(\xi)$ and $D \varphi(\xi)$ are collinear. They also have the same orientation as the sign of $d$ and $\varphi$ are the same in $U$. Thus,

$$
\mathbf{n}(\xi)^{T} D \varphi(\xi)=|\mathbf{n}(\xi)||D \varphi(\xi)| \geqslant c_{0} .
$$

The conclusion follows, if $|d(x)|\left(\tilde{\kappa}+2 \kappa c_{1}\right) \leqslant \frac{1}{2} c_{0}$.

To freely use Lemma 3.1 and Lemma 3.2, another, smaller neighborhood inside $U$ is used, which obeys the premise of Lemma 3.2 and which is such that its closure is contained in $U$. A minor additional assumption is that this neighborhood should have the form

$$
\left\{x \in \Omega|| d(x) \mid \leqslant r_{0}\right\}
$$

for some positive constant $r_{0}$. This is stronger than necessary, but simplifies the analysis. The assumption can be satisfied for all geometric configurations in which $\Gamma$ does not intersect $\partial \Omega$. To keep the notation simple, this smaller neighborhood is again called $U$. Altogether, this means

$$
|d(x)| \leqslant r_{0}<\min \left\{\frac{1}{2 \kappa}, \frac{1}{2} \frac{c_{0}}{\tilde{\kappa}+2 \kappa c_{1}}\right\} \quad \text { for all } x \in U .
$$

A useful consequence of Lemma 3.2 and $\varphi(x)=\int_{0}^{d(x)} \mathbf{n}^{T} D \varphi(p(x)+s \mathbf{n}) d s$ is

$$
|\varphi(x)| \geqslant \frac{c_{0}}{2}|d(x)| \quad \text { on } U .
$$

Up to this point, properties of the exact level set $\Gamma$ were treated. Now, $\Gamma_{h}$ is considered. 
It is assumed that the family of triangulations $\left(\mathcal{T}_{h}\right)_{h>0}$ is shape regular. $\mathbb{X}_{h}$ is the space of continuous, piecewise polynomial finite elements of degree up to $l$ on $\mathcal{T}_{h}$. We assume $\varphi_{h} \in \mathbb{X}_{h}$. A main assumption is that $\varphi_{h}$ is a quasi-optimal approximation to $\varphi$ on $U$,

$$
\left\|\varphi-\varphi_{h}\right\|_{L^{\infty}(U)} \leqslant c_{2} h^{l+1},
$$

where $c_{a}$ is a positive constant. For instance, this is satisfied, if $\varphi_{h}$ is the standard nodal interpolant of $\varphi$ and $l \leqslant k$. For simplicity, $l \leqslant k$ is assumed; otherwise, $h^{l+1}$ must be replaced by $h^{k+1}$ in (3.5). From (3.5), shape-regularity, and a standard inverse estimate, one can deduce

$$
\left\|\varphi-\varphi_{h}\right\|_{L^{\infty}(U)}+h\left\|D \varphi-D \varphi_{h}\right\|_{L^{\infty}(U)} \leqslant c_{2} h^{l+1}
$$

with some constant, which we also call $c_{2}$ for simplicity.

The first goal is to show that $\Gamma_{h}$ is the graph of a function $E$ which maps along the fibers of $p$, that is

$$
E: \Gamma \rightarrow \Gamma_{h}, \quad E(x)-x=d(E(x)) \mathbf{n}(x) \quad \text { for all } x \in \Gamma .
$$

Let the positive mesh width $h_{0}$ be given by

$$
h_{0}=\min \left\{\left(\frac{1}{2} \frac{c_{0}}{c_{2}}\right)^{1 / l},\left(\frac{1}{2} \frac{c_{0}}{c_{2}} r_{0}\right)^{1 /(l+1)}\right\}
$$

LEmma 3.3 For all $h<h_{0}$ and $x \in \partial U$, there holds $\varphi_{h}(x) d(x)>0$.

Proof. For any $x \in \partial U$, there holds $|d(x)|=r_{0}$. Obviously, $\varphi_{h}(x)=\varphi(x)-\left(\varphi(x)-\varphi_{h}(x)\right)$. From (3.4) and (3.5), one concludes for $d(x)=r_{0}$ that $\varphi_{h}(x) \geqslant \frac{1}{2} c_{0} r_{0}-c_{2} h^{l+1}$. Likewise, for $d(x)=-r_{0}$, we find $\varphi_{h}(x) \leqslant-\frac{1}{2} c_{0} r_{0}+c_{2} h^{l+1}$. The conclusion follows from $h<h_{0}$ and $c_{2} h_{0}^{l+1} \leqslant \frac{1}{2} c_{0} r_{0}$.

To proceed, we require the following representation of $\varphi_{h}$,

Lemma 3.4 For all $x \in U$, there holds

$$
\varphi_{h}(x)=\varphi_{h}(\xi)+\int_{0}^{d(x)} \mathbf{n}(\xi)^{T} D \varphi_{h}(\xi+s \mathbf{n}(\xi)) d s, \quad \xi=p(x) .
$$

Proof. Consider the line segment $L=\operatorname{conv}\{\xi, x\}$ and its intersection with the simplices in $\mathcal{T}_{h}$. In the interior of the $n$-simplices, the representation (3.9) is valid because $\varphi_{h}$ is a polynomial. In the generic case, $L$ intersects the boundaries of the $n$-simplices in a finite number of points, where $D \varphi_{h}$ has a jump discontinuity. Hence, (3.9) holds in the generic case.

In the exceptional case, $L$ intersects the boundary of some $n$-simplices $S_{j}$ in a finite number of (closed) sub-segments $L_{i}$. Now, one uses the fact that $\varphi_{h}$ is continuous and piecewise polynomial. Continuity implies, that all $S_{j}$ induce the same polynomial on the $L_{i}$. Hence, its tangential derivative on $L$ is well-defined with the exception of finitely many jump-discontinuities, and the integral formula (3.9) is valid.

Consider the approximation property (3.6) on an arbitrary (closed) $S \in \mathcal{T}_{h}, S \subset U:\left.\varphi_{h}\right|_{S}$ is a polynomial and therefore has a smooth extension to $\mathbb{R}^{n}$. The level set function $\varphi$ is $(k+1)$-times differentiable in a neighborhood of $S$. Hence, one infers from (3.6) that

$$
\max \left\{\left|D \varphi(x)-D \varphi_{h}(x)\right| \mid x \in \bar{S}\right\} \leqslant c_{2} h^{l} .
$$

An important consequence is that the $L^{\infty}$-approximation property of (3.6) is also valid on the boundaries of the simplices, where $D \varphi_{h}$ is multi-valued. 

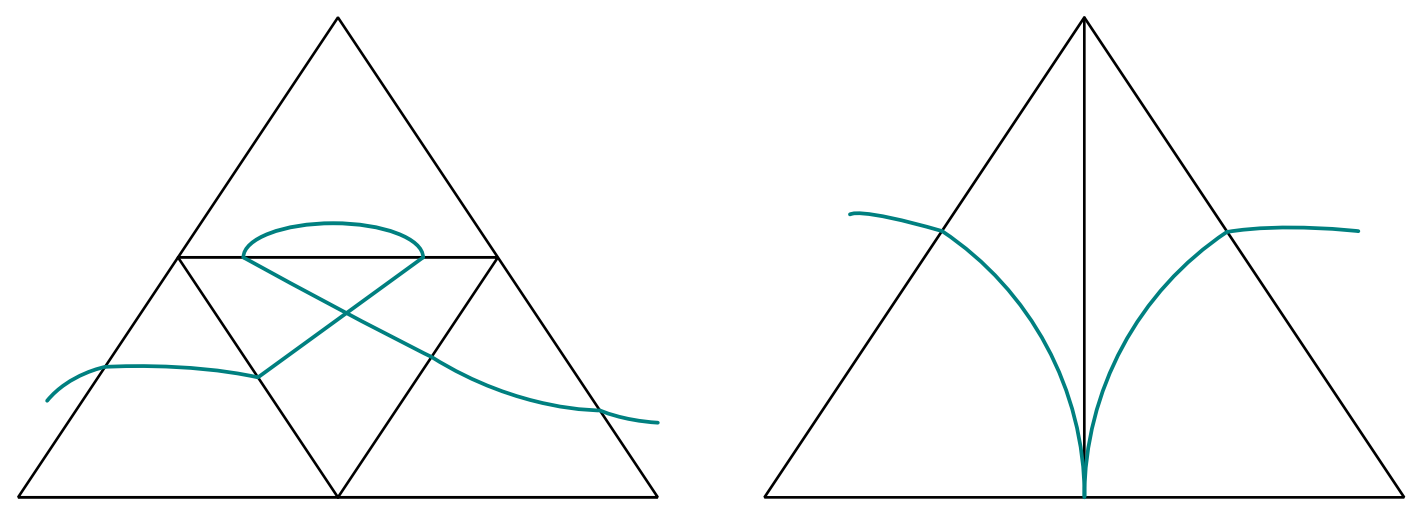

FIG. 2. Zero-levels of piecewise quadratic functions that do not occur, if (3.5) holds, cf. Remark 3.1.

Lemma 3.5 For all $h<h_{0}$ and $\xi \in \Gamma$, the function $f(r)=\varphi_{h}(\xi+r \mathbf{n}(\xi))$ is strictly increasing on $\left[-r_{0}, r_{0}\right]$.

Proof. Let $-r_{0} \leqslant s<t \leqslant r_{0}$ and $\xi \in \Gamma$ be arbitrary. Lemma 3.4 yields

$$
f(t)-f(s)=\int_{s}^{t} \mathbf{n}^{T} D \varphi_{h}(\xi+r \mathbf{n}) d r
$$

with the abbreviation $\mathbf{n}=\mathbf{n}(\xi)=\mathbf{n}(\xi+r \mathbf{n}(\xi))$. Writing $D \varphi_{h}=D \varphi-\left(D \varphi-D \varphi_{h}\right)$, Lemma 3.2 and (3.10) yield

$$
f(t)-f(s) \geqslant \frac{1}{2} c_{0}(t-s)-(t-s) c_{2} h^{l}=(t-s)\left(\frac{1}{2} c_{0}-c_{2} h^{l}\right)>0 .
$$

Lemma 3.3 and Lemma 3.5 imply that the map $E$ in (3.7) is in fact well-defined and unique, if $h<h_{0}$.

REMARK 3.1 (Geometry of $\Gamma_{h}$ ) In the interior of any $n$-simplex $S \in \mathcal{T}_{h}$, the approximate surface $\Gamma_{h} \cap S$ is a $C^{\infty}$-embedded manifold because $h<h_{0}$ and (3.6) imply $\left|D \varphi_{h}\right|>\frac{1}{2} c_{0}$. Hence, $D \varphi_{h}$ has full rank on $S$, and the implicit function theorem applies. The consequence is that singular level sets as shown on the left-hand side of Figure 2 do not occur locally on $S$.

In points $x \in \Gamma_{h} \cap S^{1} \cap S^{2}, S^{i} \in \mathcal{T}_{h}$, the approximate surface generally is not smooth. One can bound the angle between the normals to $\Gamma_{h}$ as one approaches $x$ from within $S^{i}$. By continuity, $g^{i}=\lim _{y \rightarrow x, y \in S^{i}} D \varphi_{h}(y)$ is well-defined. Let $g=D \varphi(x)$ and $\delta^{i}=g^{i}-g$. Note that the normal on $\Gamma_{h}$ in $x$ from within $S^{i}$ is $\mathbf{n}_{h}^{i}=g^{i} /\left|g^{i}\right|$. One has

$$
\mathbf{n}_{h}^{1} \mathbf{n}_{h}^{2} \geqslant 1-c h^{l}
$$

with some positive constant $c$. This implies that the angle between $\mathbf{n}_{h}^{1}$ and $\mathbf{n}_{h}^{2}$ is of order $\mathcal{O}\left(h^{l / 2}\right)$ for $h \rightarrow 0$. Hence, cusps as on the right-hand side of Figure 2 do not occur. 
To prove the statement, one uses (3.10) to find $\left|\delta^{i}\right| \leqslant c_{2} h^{l}$. Then, $\left(g^{1}\right)^{T} g^{2}=\left(g+\delta^{1}\right)^{T}(g+$ $\left.\delta^{2}\right) \geqslant|g|^{2}-c(h)$ with $c(h)=2 c_{1} c_{2} h^{l}+\left(c_{2} h^{l}\right)^{2}$. Similarly, $\left|g^{1}\right|\left|g^{2}\right| \leqslant|g|^{2}+c(h)$. Thus,

$$
\mathbf{n}_{h}^{1} \mathbf{n}_{h}^{2} \geqslant \frac{|g|^{2}-c(h)}{|g|^{2}+c(h)} \geqslant 1-\frac{2 c(h)}{|g|^{2}+c(h)} \geqslant 1-2 \frac{c(h)}{c_{0}^{2}},
$$

from which the claim follows with (3.8) and $h<h_{0}$.

The next goal is to derive the bound $\left\|d-d_{h}\right\|_{L^{\infty}(U)} \leqslant C h^{l+1}$. First, we bound the distance of $\Gamma_{h}$ to $\Gamma$. The following lemma can be found in (Reusken, 2013, La. 7.4). To make the paper self contained, we include a slightly modified proof below.

Lemma 3.6 Under (3.1), (3.3), (3.5), (3.8) and for $h<\min \left\{h_{0}, \frac{c_{0}}{\tilde{\kappa}},\left(\frac{1}{2} \frac{c_{0}^{2}}{c_{2} \tilde{\kappa}}\right)^{1 /(l+1)}\right\}$, there holds

$$
\max \left\{|d(x)| \mid x \in \Gamma_{h}\right\} \leqslant \frac{2 c_{2}}{c_{0}} h^{l+1} .
$$

Proof. Consider two arbitrary points $x, \xi \in U$ with the line segment $\operatorname{conv}\{\xi, x\} \subset U$. The Taylor expansion of $\varphi$ yields

$$
\varphi(x)=\varphi(\xi)+D \varphi(\xi)^{T}(x-\xi)+\frac{1}{2}(x-\xi)^{T} D^{2} \varphi(\zeta)(x-\xi)
$$

with some $\zeta \in \operatorname{conv}\{\xi, x\}$. Rearranging this and the Cauchy-Schwarz inequality imply

$$
\left|D \varphi(\xi)^{T}(x-\xi)\right| \leqslant|\varphi(x)-\varphi(\xi)|+\frac{1}{2} \kappa|x-\xi|^{2} .
$$

Choosing $\xi=p(x)$ yields $x-\xi=x-p(x)=d(x) \mathbf{n}(p(x))$. As $D \varphi(\xi)$ and $\mathbf{n}(\xi)$ are collinear, there holds $\left|D \varphi(\xi)^{T}(x-\xi)\right|=|D \varphi(\xi)||x-\xi|$. Furthermore, $\varphi(\xi)=0$, so

$$
c_{0}|x-\xi| \leqslant|\varphi(x)|+\frac{1}{2} \tilde{\kappa}|x-\xi|^{2} .
$$

As $\Gamma_{h} \subset U$, one can choose any $x \in \Gamma_{h}$. By adding $0=\varphi_{h}(x)$, one obtains from (3.5) that $|x-\xi|$ satisfies the quadratic inequality

$$
c_{0}|x-\xi| \leqslant c_{2} h^{l+1}+\frac{1}{2} \tilde{\kappa}|x-\xi|^{2} .
$$

Consider the corresponding quadratic equation $\frac{1}{2} \tilde{\kappa}|x-\xi|^{2}-c_{0}|x-\xi|+c_{2} h^{l+1}=0$, which has a global minimum at $\frac{c_{0}}{\tilde{\kappa}}$. By the premises, one knows $0 \leqslant|x-\xi| \leqslant \frac{c_{0}}{\tilde{\kappa}}$. Therefore,

$$
|x-\xi| \leqslant \frac{2 \frac{c_{2}}{\tilde{\kappa}} h^{l+1}}{\frac{c_{0}}{\tilde{\kappa}}+\sqrt{\left(\frac{c_{0}}{\tilde{\kappa}}\right)^{2}-2 \frac{c_{2}}{\tilde{\kappa}} h^{l+1}}} \leqslant 2 \frac{c_{2}}{c_{0}} h^{l+1} .
$$

The preceding result easily yields a one-sided estimate on $U$,

LEMma 3.7 Under the assumptions of Lemma 3.6, the inequality $|d(x)|-\left|d_{h}(x)\right| \leqslant 2 \frac{c_{2}}{c_{0}} h^{l+1}$ holds for all $x \in U$. 


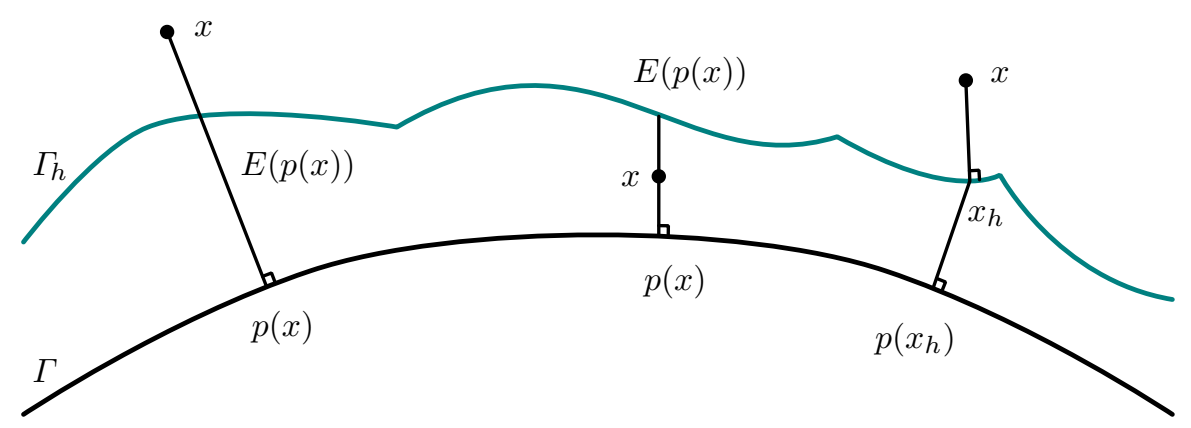

Fig. 3. Notation in proofs, from left to right: Lemma 3.9, Theorem 3.1, Lemma 3.7.

Proof. Let $x \in U$ be an arbitrary point. By the closedness of $\Gamma_{h}$, there exists a point $x_{h} \in \Gamma_{h}$ of minimal distance, $\left|x-x_{h}\right|=\min \left\{|x-y| \mid y \in \Gamma_{h}\right\}=\left|d_{h}(x)\right|$. The distance of $x$ to $\Gamma$ satisfies $|d(x)|=\min \{|x-y| \mid y \in \Gamma\} \leqslant\left|x-p\left(x_{h}\right)\right|$. The triangle inequality implies

$$
|d(x)|-\left|d_{h}(x)\right| \leqslant\left|x-p\left(x_{h}\right)\right|-\left|x-x_{h}\right| \leqslant\left|x_{h}-p\left(x_{h}\right)\right| \leqslant 2 \frac{c_{2}}{c_{0}} h^{l+1},
$$

where Lemma 3.6 is used in the final step.

Next, Lemma 3.7 is established with $d$ and $d_{h}$ exchanged. If $\varphi_{h}$ were smooth, one could appeal to the symmetry of the situation to obtain the desired result. But the proof of Lemma 3.6 fails for $\varphi_{h}$. Instead, the proof relies on the key property of the map $E$ that it maps $\Gamma \rightarrow \Gamma_{h}$ along the fibers of $p$. The first step is the analogue of Lemma 3.6,

LEMma 3.8 Under the assumptions of Lemma 3.6, $\max \left\{\left|d_{h}(\xi)\right| \mid \xi \in \Gamma\right\} \leqslant \frac{2 c_{2}}{c_{0}} h^{l+1}$.

Proof. Take any $\xi \in \Gamma$. Then, $\left|d_{h}(\xi)\right| \leqslant|\xi-E(\xi)|$. Due to (3.7), one can express the latter as $|d(E(\xi)) \mathbf{n}(\xi)|$, to which Lemma 3.6 can be applied.

From Lemma 3.6, one obtains the final one-sided estimate,

LEMma 3.9 Under the assumptions of Lemma 3.6, the inequality $\left|d_{h}(x)\right|-|d(x)| \leqslant 2 \frac{c_{2}}{c_{0}} h^{l+1}$ holds for all $x \in U$.

Proof. Let $x \in U$ be an arbitrary point and $\xi=p(x)$. Then, $|d(x)|=|x-\xi|$. The distance of $\xi \in \Gamma$ to $\Gamma_{h}$ satisfies $\left|d_{h}(\xi)\right|=\min \left\{\left|\xi-x_{h}\right| \mid x_{h} \in \Gamma_{h}\right\} \leqslant|\xi-E(\xi)|$. The triangle inequality implies

$$
\left|d_{h}(x)\right|-|d(x)| \leqslant|x-E(\xi)|-|x-\xi| \leqslant|\xi-E(\xi)| .
$$

As in the preceding proof, one uses (3.7) to bound the final term by $|d(E(\xi)) \mathbf{n}(\xi)|$, to which Lemma 3.6 can be applied.

Theorem 3.1 Under the assumptions of Lemma 3.6, there holds

$$
\left\|d-d_{h}\right\|_{L^{\infty}(U)} \leqslant 2 \frac{c_{2}}{c_{0}} h^{l+1} .
$$

Proof. Take any $x \in U$. If $d(x)$ and $d_{h}(x)$ have the same sign, $\left|d(x)-d_{h}(x)\right|=|| d(x)|-| d_{h}(x)||$ and the claim follows from Lemma 3.7 and Lemma 3.9.

Otherwise, the signs are different and $x$ lies on the line segment between $\xi=p(x) \in \Gamma$ and $x_{h}=E(\xi) \in \Gamma_{h}$, cf. Figure 3. Hence, $|x-\xi|+\left|x_{h}-x\right|=\left|x_{h}-\xi\right|$. Clearly, $\left|d_{h}(x)\right| \leqslant\left|x_{h}-x\right|$. 
One obtains

$$
\left|d(x)-d_{h}(x)\right|=|d(x)|+\left|d_{h}(x)\right| \leqslant|x-\xi|+\left|x_{h}-x\right|=\left|x_{h}-\xi\right|,
$$

from which the claim follows with Lemma 3.6.

We turn to the approximate distance function $\tilde{d}_{h}$ and its zero level $\tilde{\Gamma}_{h}$, which are the output of the redistancing algorithm. The standard nodal interpolation operators $I_{h}$ on $\mathcal{T}_{h}^{\Gamma}$ are a stable family because of shape-regularity. This means

$$
\left\|I_{h} f\right\|_{L^{\infty}\left(\Omega_{h}^{\Gamma}\right)} \leqslant c_{S}\|f\|_{L^{\infty}\left(\Omega_{h}^{\Gamma}\right)} \quad \text { for all } f \in C(U)
$$

for some positive constant $c_{S}$. Due to the $C^{k+1}$-smoothness of $d$, there holds $\left\|I_{h} d-d\right\|_{L^{\infty}\left(\Omega_{h}^{\Gamma}\right)} \leqslant$ $c_{I}\|d\|_{C^{l+1}(U)} h^{l+1}$ for some positive constant $c_{I}$.

THEOREM 3.2 Under the assumptions of Lemma 3.6, there holds

$$
\left\|\tilde{d}_{h}-d\right\|_{L^{\infty}\left(\Omega_{h}^{\Gamma}\right)} \leqslant c h^{l+1}, \quad\left\|\tilde{d}_{h}-d_{h}\right\|_{L^{\infty}\left(\Omega_{h}^{\Gamma}\right)} \leqslant c h^{l+1}
$$

with the constant $c=\left(2 \frac{c_{2}}{c_{0}}\left(1+c_{S}\right)+c_{I}\|d\|_{C^{l+1}(U)}\right)$.

Proof. Writing $\tilde{d}_{h}-d=\tilde{d}_{h}-I_{h} d+I_{h} d-d$, the stability of $\left(I_{h}\right)_{h>0}$ and Theorem 3.1 yield

$$
\left\|\tilde{d}_{h}-d\right\|_{L^{\infty}\left(\Omega_{h}^{\Gamma}\right)} \leqslant c_{S} \cdot 2 \frac{c_{2}}{c_{0}} h^{l+1}+c_{I}\|d\|_{C^{l+1}(U)} h^{l+1} .
$$

The other inequality follows from this one and Theorem 3.1 via $\tilde{d}_{h}-d_{h}=\tilde{d}_{h}-d-\left(d_{h}-d\right)$.

The most straightforward application of Theorem 3.2 is to bound the Hausdorff distance between $\tilde{\Gamma}_{h}$ and $\Gamma$, respectively $\Gamma_{h}$. For two sets $A, B$ with (unsigned) distance functions $d_{A}$ and $d_{B}$, their Hausdorff distance is

$$
\operatorname{dist}(A, B)=\max \left\{\max \left\{d_{B}(x) \mid x \in A\right\}, \max \left\{d_{A}(x) \mid x \in B\right\}\right\} .
$$

Corollary 3.1 Under the assumptions of Lemma 3.6, there holds

$$
\operatorname{dist}\left(\tilde{\Gamma}_{h}, \Gamma\right) \leqslant c h^{l+1}, \quad \operatorname{dist}\left(\tilde{\Gamma}_{h}, \Gamma_{h}\right) \leqslant c h^{l+1}
$$

with the constant $c$ of Theorem 3.2.

Proof. The first inequality follows from the first inequality of Theorem 3.2 by noting that $d$ is zero on $\Gamma$ and conversely $\tilde{d}_{h}$ is zero on $\tilde{\Gamma}_{h}$. The second inequality follows in the same manner from the second inequality of Theorem 3.2.

The unit-length vector field $\tilde{\mathbf{n}}_{h}=D \tilde{d}_{h} /\left|D \tilde{d}_{h}\right|$ on $\Omega_{h}^{\Gamma}$ is an approximation of $\mathbf{n}$,

COROLlary 3.2 Under the assumptions of Lemma 3.6, there holds

$$
\left\|\tilde{\mathbf{n}}_{h}-\mathbf{n}\right\|_{L^{\infty}\left(\Omega_{h}^{\Gamma}\right)} \leqslant c h^{l}
$$

with some positive constant $c$.

Proof. As $\tilde{d}_{h} \in \mathbb{X}_{h}$, one can apply a standard inverse estimate to the first inequality in Theorem 3.2 to produce

$$
\left\|D \tilde{d}_{h}-D d\right\|_{L^{\infty}\left(\Omega_{h}^{\Gamma}\right)} \leqslant c h^{l}
$$



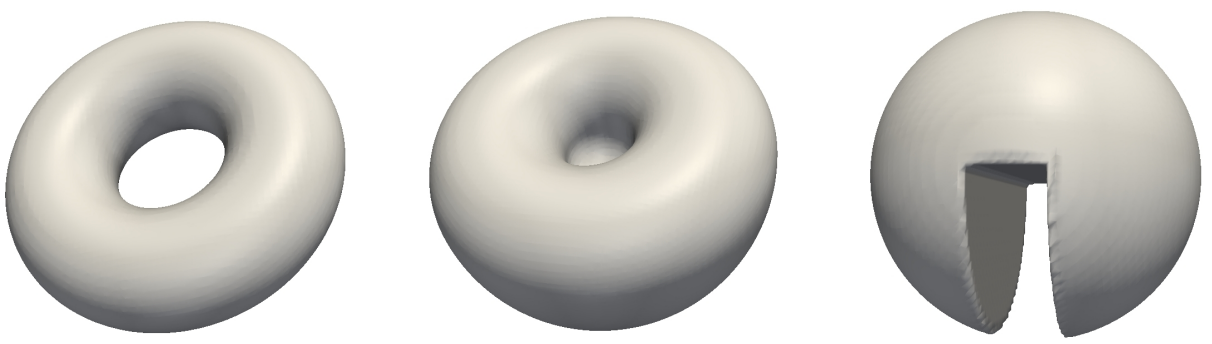

FIG. 4. Test cases: Torus, apple, and slotted sphere.

with some positive constant $c$. From this and the triangle inequality, one gets ||$\left|D \tilde{d}_{h}\right|-$ $1\left\|_{L^{\infty}\left(\Omega_{h}^{\Gamma}\right)} \leqslant\right\| D \tilde{d}_{h}-\mathbf{n} \|_{L^{\infty}\left(\Omega_{h}^{\Gamma}\right)} \leqslant c h^{l}$. Writing

$$
\tilde{\mathbf{n}}_{h}-\mathbf{n}=\tilde{\mathbf{n}}_{h}\left(1-\left|D \tilde{d}_{h}\right|\right)+D \tilde{d}_{h}-\mathbf{n} \quad \text { a.e. on } \Omega_{h}^{\Gamma},
$$

the conclusion follows by taking the $L^{\infty}\left(\Omega_{h}^{\Gamma}\right)$-norm on both sides.

REMARK 3.2 Surfaces with edges do not satisfy the assumption $\varphi \in C^{k+1}(U)$ for any positive integer $k$. However, there may be subsets of $U$ of the form $\left\{x+s \mathbf{n}(x)|x \in \gamma, 0 \leqslant| s \mid \leqslant r_{0}\right\}, \gamma \subset \Gamma$, on which $\varphi$ is $C^{k+1}$-smooth. The preceding analysis can be modified to show that Theorem 3.2 and Corollary 3.2 hold on these subsets.

\section{Implementation}

The method is implemented for piecewise linear and piecewise quadratic finite elements in the 3D finite element code Drops, Groß et al. (2002). The real roots of the univariate polynomial (2.3), which has degree up to 6 , are computed with a standard method: They are isolated in disjoint intervals with a method based on Sturm-sequences. Within an interval, the final value is computed with the Anderson-Björk-method Anderson \& Björck (1973) which is a bracketing method. The roots are located in intervals of size less then 1e-12. The Schur decomposition of the matrix $A$ is computed with Jacobi's method Golub \& Van Loan (1996), in which the root of the sum-of-squares of the off-diagonal entries is reduced below 1e-12.

The computations of the values of $\tilde{d}_{h}(x)$ for different degrees of freedom $x \in \mathcal{N}_{h}$ are completely independent of each other. Therefore, the redistancing method is well-suited for a shared-memory parallelization. An OpenMP-based parallelization has been implemented and is used for the experiments in Section 5. Almost perfect speedup is observed.

\section{Numerical experiments}

Three test cases are considered: A torus, an 'apple', and a slotted sphere, which are all embedded in the cube $\Omega=(-1,1)^{3}$. The surfaces are displayed in Figure 4. The torus is $C^{\infty}$-embedded, hence, Theorem 3.2 applies without restrictions. The apple is composed of two half-spheres with different radii and a slice of a torus connecting them. Each of these pieces 

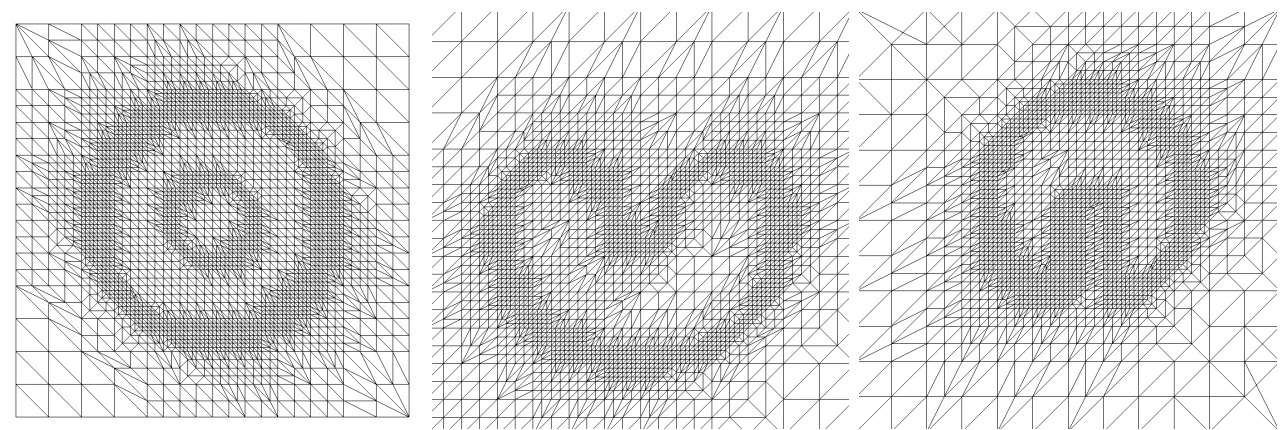

FIG. 5. Slices of $\mathcal{T}_{h}, \ell=4$, for the torus, the apple, and the slotted sphere.
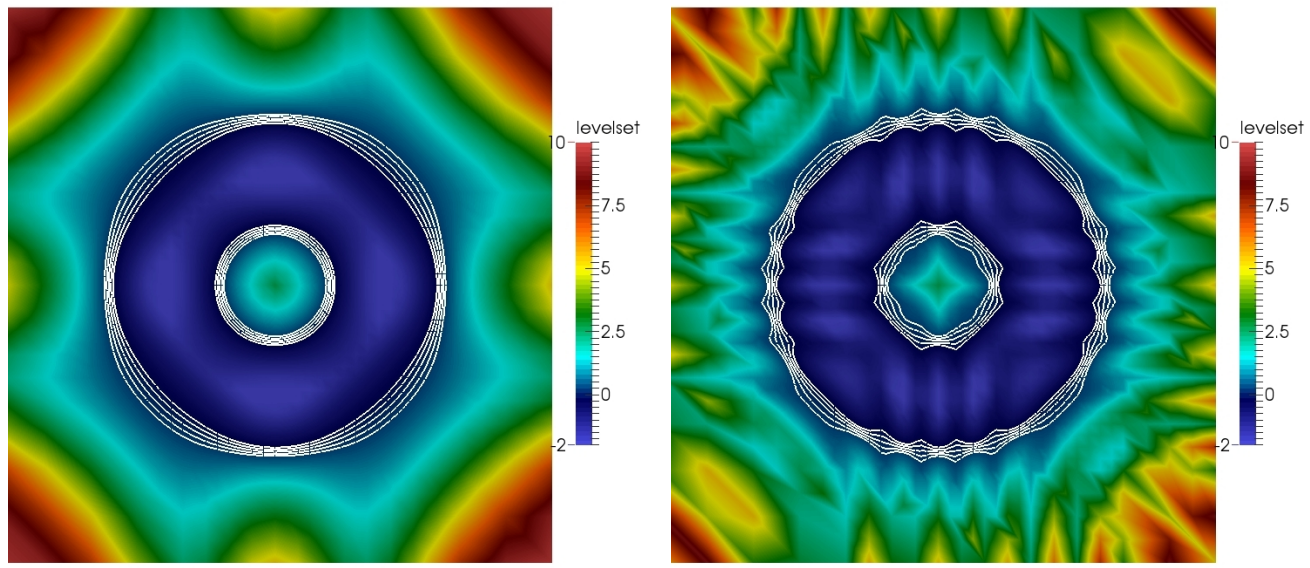

Fig. 6. Torus: Values of $\varphi^{\alpha}$ and level sets of $0, \pm 0.1, \pm 0.2$ for $\alpha \in\{10,50\}$.

is $C^{\infty}$-embedded, but the radius of the osculating sphere has a jump discontinuity where the half-spheres meet the torus. Therefore, the apple is globally only $C^{1}$-embedded. It is used in Reusken (2013) as an example of a mildly rough surface. The slotted sphere is piecewise smooth, but at the edges the embedding is not even $C^{1}$. For all three surfaces, the signed distance function $d$ is available via an explicit formula.

To generate the triangulations $\mathcal{T}_{h}$, the domain is subdivided uniformly into $3 \times 3 \times 3$ smaller cubes. Each of these is partitioned into 6 tetrahedra which yields the Kuhn-triangulation $\mathcal{T}_{h_{0}}$ with mesh width $h_{0}=\frac{2}{3}$. Local red-green refinement is applied in the vicinity of $\Gamma$ until all tetrahedra of $\mathcal{T}_{h}$ intersecting $\Gamma$ have the mesh-width $h=h(\ell)=2^{-\ell} h_{0}, \ell \in\{2, \ldots, 7\}$. Slices of $\mathcal{T}_{h}$ for $\ell=4$ are shown in Figure 5 . The space of continuous, piecewise quadratic finite elements is used for the discrete level set functions $\varphi_{h}$. Let $I_{h}$ be the standard nodal interpolation operator of this space on $\mathcal{T}_{h}$, and let $\mathcal{N}_{h}$ be the set of vertices and edge-barycenters of $\mathcal{T}_{h}^{\Gamma}$. As 


\begin{tabular}{lcccccc}
\hline & \multicolumn{2}{c}{$\alpha=1$} & \multicolumn{2}{c}{$\alpha=10$} & \multicolumn{2}{c}{$\alpha=50$} \\
$\ell$ & $e_{\infty}$ & order & $e_{\infty}$ & order & $e_{\infty}$ & order \\
\hline 2 & $7.57 \mathrm{e}-3$ & & $5.60 \mathrm{e}-3$ & & $4.49 \mathrm{e}-2$ & \\
3 & $5.75 \mathrm{e}-4$ & 3.72 & $8.27 \mathrm{e}-4$ & 2.76 & $1.66 \mathrm{e}-2$ & 1.43 \\
4 & $7.80 \mathrm{e}-5$ & 2.88 & $1.21 \mathrm{e}-4$ & 2.77 & $3.33 \mathrm{e}-3$ & 2.32 \\
5 & $1.08 \mathrm{e}-5$ & 2.86 & $1.56 \mathrm{e}-5$ & 2.96 & $5.20 \mathrm{e}-4$ & 2.68 \\
6 & $1.42 \mathrm{e}-6$ & 2.92 & $1.87 \mathrm{e}-6$ & 3.06 & $6.89 \mathrm{e}-5$ & 2.92 \\
7 & $1.73 \mathrm{e}-7$ & 3.04 & $2.45 \mathrm{e}-7$ & 2.94 & $8.78 \mathrm{e}-6$ & 2.97 \\
\hline
\end{tabular}

Table 1. Torus: Error and order of convergence.

\begin{tabular}{cccccccc}
\hline & \multicolumn{2}{c}{$\alpha=1$} & \multicolumn{3}{c}{$\alpha=10$} & \multicolumn{2}{c}{$\alpha=50$} \\
$\ell$ & $e_{\infty}^{D}$ & order & $e_{\infty}^{D}$ & order & $e_{\infty}^{D}$ & order & $\tilde{e}_{\infty}^{D}$ \\
\hline 2 & $2.13 \mathrm{e}-1$ & & $2.03 \mathrm{e}-1$ & & $2.89 \mathrm{e}-1$ & & $2.17 \mathrm{e}-1$ \\
3 & $1.66 \mathrm{e}-2$ & 3.69 & $1.77 \mathrm{e}-2$ & 3.52 & $1.46 \mathrm{e}-1$ & 0.98 & $1.59 \mathrm{e}-2$ \\
4 & $3.46 \mathrm{e}-3$ & 2.26 & $3.85 \mathrm{e}-3$ & 2.20 & $6.44 \mathrm{e}-2$ & 1.18 & $2.96 \mathrm{e}-3$ \\
5 & $8.70 \mathrm{e}-4$ & 1.99 & $1.11 \mathrm{e}-3$ & 1.80 & $1.99 \mathrm{e}-2$ & 1.69 & $7.69 \mathrm{e}-4$ \\
6 & $2.13 \mathrm{e}-4$ & 2.03 & $3.10 \mathrm{e}-4$ & 1.83 & $5.90 \mathrm{e}-3$ & 1.75 & $1.84 \mathrm{e}-4$ \\
7 & $5.31 \mathrm{e}-5$ & 1.99 & $7.33 \mathrm{e}-5$ & 2.08 & $1.57 \mathrm{e}-3$ & 1.91 & $4.49 \mathrm{e}-5$ \\
\hline
\end{tabular}

Table 2. Torus: Gradient-error and order of convergence.

exact level set functions, the perturbations of $d$

$$
\varphi^{(\alpha)}=q^{(\alpha)} d \quad \text { with } q^{(\alpha)}(x)=9.0+4.0 \cos \left(\alpha \frac{x_{1} x_{2}}{|x|}\right), \quad \alpha \in\{1,10,50\}
$$

are taken. The functions $\varphi^{\alpha}$ have the same zero level as $d$, and they have a large gradient. The parameter $\alpha$ determines the amount of distortion and the oscillations of the gradient. For $\alpha \in\{10,50\}$, Figure 6 shows the level sets of $0, \pm 0.1, \pm 0.2$ of $\varphi^{(\alpha)}$ for the torus at the cross section displayed in Figure 5. The input of the redistancing method is the nodal interpolant $\varphi_{h}^{\alpha}=I_{h} \varphi^{\alpha}$.

As error measure, the maximum norm error between $\tilde{d}_{h}$ and $d$ in the degrees of freedom is used,

$$
e_{\infty}:=\max _{x \in \mathcal{N}_{h}}\left|\tilde{d}_{h}(x)-d(x)\right|
$$

This can be compared to the error bounds of Theorem 3.2. Furthermore, the gradient of $\tilde{d}_{h}$ in $\Omega_{h}^{\Gamma}$ is considered,

$$
e_{\infty}^{D}:=\max _{T \in \mathcal{T}_{h}^{\Gamma}}\left|\sqrt{\frac{1}{|T|} \int_{T}\left|D \tilde{d}_{h}(s)\right|^{2} d s}-1\right|,
$$

which can be compared to (3.12) and Corollary 3.2. We also compute this error measure with $\tilde{d}_{h}$ replaced by $I_{h} d$ to provide a reference for the absolute magnitude of $e_{\infty}^{D}$. This is referred to as $\tilde{e}_{\infty}^{D}$ below. 


\begin{tabular}{cccccc}
\hline$\ell$ & $e_{\infty}$ & order & $e_{\infty}^{D}$ & order & $\tilde{e}_{\infty}^{D}$ \\
\hline 2 & $2.05 \mathrm{e}-2$ & & $1.42 \mathrm{e}-1$ & & $1.12 \mathrm{e}-1$ \\
3 & $3.32 \mathrm{e}-3$ & 2.63 & $4.93 \mathrm{e}-2$ & 1.53 & $3.41 \mathrm{e}-2$ \\
4 & $4.43 \mathrm{e}-4$ & 2.90 & $9.47 \mathrm{e}-3$ & 2.38 & $7.08 \mathrm{e}-3$ \\
5 & $5.78 \mathrm{e}-5$ & 2.94 & $2.33 \mathrm{e}-3$ & 2.02 & $1.74 \mathrm{e}-3$ \\
6 & $7.45 \mathrm{e}-6$ & 2.96 & $6.10 \mathrm{e}-4$ & 1.93 & $3.83 \mathrm{e}-4$ \\
7 & $9.35 \mathrm{e}-7$ & 2.99 & $1.54 \mathrm{e}-4$ & 1.99 & $9.53 \mathrm{e}-5$ \\
\hline
\end{tabular}

Table 3. Apple: Error, gradient-error, and order of convergence for $\alpha=10$.

\begin{tabular}{cccccc}
\hline$\ell$ & $e_{\infty}$ & order & $e_{\infty}^{D}$ & order & $\tilde{e}_{\infty}^{D}$ \\
\hline 2 & $4.45 \mathrm{e}-2$ & - & $3.63 \mathrm{e}-1$ & - & $3.83 \mathrm{e}-1$ \\
3 & $2.22 \mathrm{e}-2$ & 1.01 & $3.42 \mathrm{e}-1$ & 0.09 & $4.16 \mathrm{e}-1$ \\
4 & $1.18 \mathrm{e}-2$ & 0.91 & $3.54 \mathrm{e}-1$ & -0.05 & $6.18 \mathrm{e}-1$ \\
5 & $4.93 \mathrm{e}-3$ & 1.26 & $3.45 \mathrm{e}-1$ & 0.04 & $3.70 \mathrm{e}-1$ \\
6 & $5.01 \mathrm{e}-3$ & -0.02 & $4.14 \mathrm{e}-1$ & -0.26 & $3.71 \mathrm{e}-1$ \\
7 & $3.44 \mathrm{e}-3$ & 0.54 & $1.26 \mathrm{e} 0$ & -1.61 & $3.70 \mathrm{e}-1$ \\
\hline
\end{tabular}

Table 4. Slotted sphere: Error, gradient-error, and order of convergence for $\alpha=10$.

The results for the torus are shown in the Tables 1,2 . One can observe order 3 for $e_{\infty}$ for all values of $\alpha$. This agrees with the bound in Theorem 3.2. For stronger perturbations, the convergence sets in on finer meshes. For $e_{\infty}^{D}$, order 2 is observed. For all values $\alpha \in\{1,10,50\}$, the redistancing method produces results on the very coarse meshes $\mathcal{T}_{h}$ with $\ell \in\{0,1\}$. In this sense, it is a robust method. This is in contrast to the iterative method of Reusken (2013), where convergence problems are reported for $\alpha=50$ up to level 5 .

Table 3 contains the errors for the apple for $\alpha=10$. The results for $\alpha \in\{1,50\}$ are similar and are not displayed separately. Beginning with $\ell=4$, order 3 is observed for $e_{\infty}$ and order 2 for $e_{\infty}^{D}$. This is better than predicted by Theorem 3.2 as the embedding of the geometry is not globally $C^{3}$-smooth.

In the final experiment, the slotted sphere is considered. The errors for $\alpha=10$ are shown in Table 4 . The global error measure $e_{\infty}$ decreases only marginally as $\ell$ is increased, and $e_{\infty}^{D}$ even increases. This is in agreement with the fact that the slotted sphere is not smoothly embedded at the edges of the slot.

As explained in Remark 3.2, the large errors are confined to the vicinity of the edges. A visual comparison of the zero levels of $I_{h} d$ and $\tilde{d}_{h}$, cf. Figure 7 , indicates that this is the case. Let $d_{e}$ be the unsigned distance-function for the edges of the slotted sphere. A tedious, elementary computation yields an explicit expression for this function. It is used to define the local error away from the edges of the geometry,

$$
\hat{e}_{\infty}:=\max \left\{\left|\tilde{d}_{h}(x)-d(x)\right| \mid x \in \mathcal{N}_{h}, d_{e}(x)>3 h\right\} .
$$

Furthermore, $\hat{e}_{\infty}^{D}$ and $\hat{\tilde{e}}_{\infty}^{D}$ are defined like $e_{\infty}^{D}$ and $\tilde{e}_{\infty}^{D}$, but a tetrahedron $T \in \mathcal{T}_{h}$ is only considered, if its barycenter $x$ satisfies $d_{e}(x)>3 h$. 

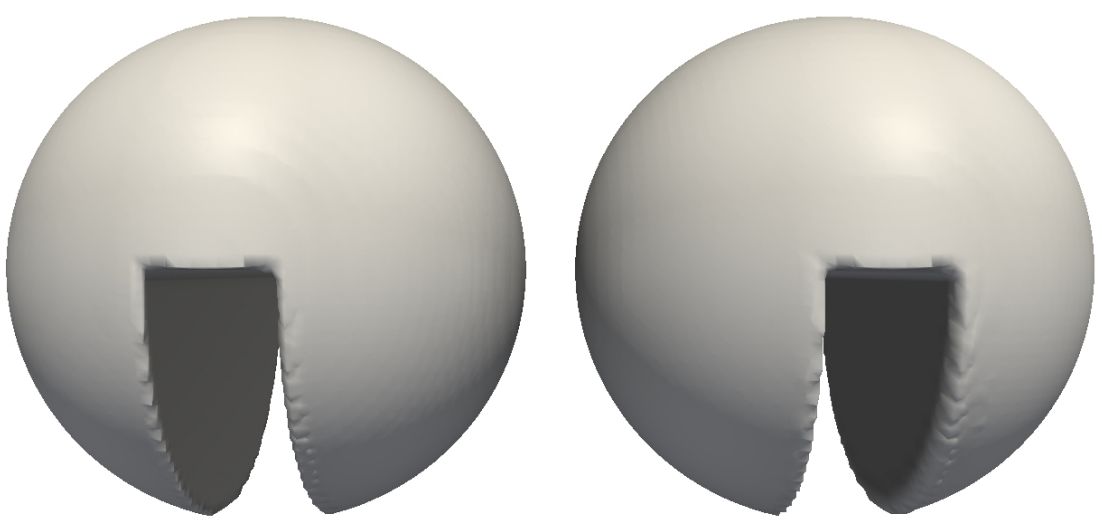

FIG. 7. Slotted sphere: Zero level of $I_{h} d$ (left) and of $\tilde{d}_{h}$ (right).

\begin{tabular}{lcccccc}
\hline & \multicolumn{2}{c}{$\alpha=1$} & \multicolumn{2}{c}{$\alpha=10$} & \multicolumn{2}{c}{$\alpha=50$} \\
$\ell$ & $\hat{e}_{\infty}$ & order & $\hat{e}_{\infty}$ & order & $\hat{e}_{\infty}$ & order \\
\hline 2 & $9.23 \mathrm{e}-4$ & & $4.17 \mathrm{e}-3$ & & $3.85 \mathrm{e}-2$ & \\
3 & $9.81 \mathrm{e}-5$ & 3.23 & $6.10 \mathrm{e}-4$ & 2.77 & $1.39 \mathrm{e}-2$ & 1.47 \\
4 & $1.28 \mathrm{e}-5$ & 2.93 & $9.58 \mathrm{e}-5$ & 2.67 & $2.24 \mathrm{e}-3$ & 2.63 \\
5 & $1.72 \mathrm{e}-6$ & 2.90 & $1.26 \mathrm{e}-5$ & 2.93 & $3.03 \mathrm{e}-4$ & 2.89 \\
6 & $2.22 \mathrm{e}-7$ & 2.95 & $1.59 \mathrm{e}-6$ & 2.98 & $3.82 \mathrm{e}-5$ & 2.99 \\
7 & $2.92 \mathrm{e}-8$ & 2.93 & $1.98 \mathrm{e}-7$ & 3.01 & $4.84 \mathrm{e}-6$ & 2.98 \\
\hline
\end{tabular}

Table 5. Slotted sphere: Error and order of convergence away from the edges.

\begin{tabular}{cccccccc}
\hline & \multicolumn{2}{c}{$\alpha=1$} & \multicolumn{3}{c}{$\alpha=10$} & \multicolumn{2}{c}{$\alpha=50$} \\
$\ell$ & $\hat{e}_{\infty}^{D}$ & order & $\hat{e}_{\infty}^{D}$ & order & $\hat{e}_{\infty}^{D}$ & order & $\hat{\tilde{e}}_{\infty}^{D}$ \\
\hline 2 & $4.84 \mathrm{e}-3$ & & $1.19 \mathrm{e}-2$ & & $1.16 \mathrm{e}-1$ & & $4.25 \mathrm{e}-3$ \\
3 & $2.22 \mathrm{e}-3$ & 1.12 & $4.57 \mathrm{e}-3$ & 1.38 & $1.18 \mathrm{e}-1$ & -0.02 & $1.90 \mathrm{e}-3$ \\
4 & $6.17 \mathrm{e}-4$ & 1.85 & $1.29 \mathrm{e}-3$ & 1.82 & $4.25 \mathrm{e}-2$ & 1.47 & $4.45 \mathrm{e}-4$ \\
5 & $1.56 \mathrm{e}-4$ & 1.98 & $4.05 \mathrm{e}-4$ & 1.68 & $1.19 \mathrm{e}-2$ & 1.83 & $1.08 \mathrm{e}-4$ \\
6 & $3.72 \mathrm{e}-5$ & 2.07 & $1.07 \mathrm{e}-4$ & 1.92 & $3.08 \mathrm{e}-3$ & 1.95 & $2.68 \mathrm{e}-5$ \\
7 & $9.62 \mathrm{e}-6$ & 1.95 & $2.56 \mathrm{e}-5$ & 2.06 & $7.84 \mathrm{e}-4$ & 1.97 & $6.62 \mathrm{e}-6$ \\
\hline
\end{tabular}

Table 6. Slotted sphere: Gradient-error and order of convergence away from the edges. 
The resulting errors are displayed in Table 5 and Table 6 . One clearly observes order 3 for $\hat{e}_{\infty}$ and order 2 for $\hat{e}_{\infty}^{D}$.

\section{Acknowledgments}

The authors thank Arnold Reusken for reading and discussing the initial version of this paper. This lead to an improved presentation.

\section{References}

Anderson, N. \& BJÖRCK, A. (1973) A new high order method of regula falsi type for computing a root of an equation. Nordisk Tidskr. Informationsbehandling (BIT), 13, 253264.

Ausas, R. F., Dari, E. A. \& Buscaglia, G. C. (2011) A geometric mass-preserving redistancing scheme for the level set function. International Journal for Numerical Methods in Fluids, 65, 989-1010.

Chopp, D. L. (2001) Some improvements of the fast marching method. SIAM J. Sci. Comput., 23, 230-244 (electronic).

Cox, D., Little, J. \& O'SheA, D. (1997) Ideals, varieties, and algorithms. Undergraduate Texts in Mathematics, second edn. New York: Springer-Verlag, pp. xiv+536. An introduction to computational algebraic geometry and commutative algebra.

Gilbarg, D. \& Trudinger, N. S. (1977) Elliptic partial differential equations of second order. Berlin: Springer-Verlag, pp. $\mathrm{x}+401$. Grundlehren der Mathematischen Wissenschaften, Vol. 224.

Golub, G. H. \& VAN Loan, C. F. (1996) Matrix computations. Johns Hopkins Studies in the Mathematical Sciences, third edn. Baltimore, MD: Johns Hopkins University Press, pp. $\mathrm{xxx}+698$

Gross, S., Peters, J., Reichelt, V. \& Reusken, A. (2002) The DROPS Package for Numerical Simulations of Incompressible Flows Using Parallel Adaptive Multigrid Techniques. IGPM-Report 211. IGPM, RWTH Aachen University.

Hartmann, D., Meinke, M. \& Schröder, W. (2010) The constrained reinitialization equation for level set methods. Journal of Computational Physics, 229, 1514 - 1535.

Kimmel, R. \& Sethian, J. A. (1998) Computing geodesic paths on manifolds. Proc. Natl. Acad. Sci. USA, 95, 8431-8435 (electronic).

Kimmel, R. \& Sethian, J. A. (2001) Optimal algorithm for shape from shading and path planning. Journal of Mathematical Imaging and Vision, 14, 237-244.

Mut, F., Buscaglia, G. C. \& Dari, E. A. (2006) New mass-conserving algorithm for level set redistancing on unstructured meshes. Journal of Applied Mechanics, 73, 1011-1016.

Osher, S. \& FEDKIw, R. P. (2001) Level set methods: An overview and some recent results. Journal of Computational Physics, 169, 463 - 502. 
Reusken, A. (2013) A finite element level set redistancing method based on gradient recovery. SIAM J. Numer. Anal., 51, 2723-2745.

Sethian, J. A. (1996) A fast marching level set method for monotonically advancing fronts. Proc. Nat. Acad. Sci. U.S.A., 93, 1591-1595.

Sethian, J. A. (1999) Level set methods and fast marching methods. Cambridge Monographs on Applied and Computational Mathematics, vol. 3, second edn. Cambridge: Cambridge University Press, pp. xx+378.

Sussman, M., Smereka, P. \& Osher, S. (1994) A level set approach for computing solutions to incompressible two-phase flow. Journal of Computational Physics, 114, 146-159. 